\title{
Characterizations of Nonlinear Lie Derivations of $B(X)$
}

\author{
Donghua Huo, ${ }^{1,2}$ Baodong Zheng, ${ }^{1}$ and Hongyu Liu ${ }^{2}$ \\ ${ }^{1}$ Department of Mathematics, Harbin Institute of Technology, Harbin 150001, China \\ ${ }^{2}$ Department of Mathematics, Mudanjiang Normal College, Mudanjiang 157012, China
}

Correspondence should be addressed to Baodong Zheng; zbd@hit.edu.cn

Received 25 December 2012; Accepted 3 February 2013

Academic Editor: Chunrui Zhang

Copyright (C) 2013 Donghua Huo et al. This is an open access article distributed under the Creative Commons Attribution License, which permits unrestricted use, distribution, and reproduction in any medium, provided the original work is properly cited.

Let $X$ be an infinite dimensional Banach space, and $\Phi: B(X) \rightarrow B(X)$ is a nonlinear Lie derivation. Then $\Phi$ is the form $\delta+\tau$ where $\delta$ is an additive derivation of $B(X)$ and $\tau$ is a map from $B(X)$ into its center $Z_{B(X)}$, which maps commutators into the zero.

\section{Introduction}

The local actions of derivations of operator algebras are still not completely understood. One is the well-known local derivation problem. The other is to study conditions under derivations of operator algebras that can be completely determined by the action on some sets of operators. In recent years there has been a great interest in the study of Lie derivations of operator algebras (e.g., see [1-6]). In particular, the structure of additive or linear Lie derivation on ring or algebra has been studied by many authors (e.g., see [7-10]).

Let $X$ be a Banach space and let $B(X)$ be the algebra of all bounded linear operators on $X$. Some progress has been made towards understanding the Lie derivation recently. In [11], it was shown that letting $X$ be a Banach space of dimension greater than 2, if $\delta: B(X) \rightarrow B(X)$ is a linear map satisfying $\delta([A, B])=[\delta(A), B]+[A, \delta(B)]$ for any $A, B \in B(X)$ with $A B=0$, then $\delta=d+\tau$, where $d$ is a derivation of $B(X)$ and $\tau: B(X) \rightarrow C I$ is a linear map vanishing at commutators $[A, B]$ with $A B=0$. In [12], it was shown that every nonlinear Lie derivation of triangular algebras is the sum of an additive derivation and a map into its center sending commutators to zero. Ji and Qi [13] proved that letting $\mathscr{T}$ be a triangular algebra over a commutative ring $R$, if $\delta: \mathscr{T} \rightarrow \mathscr{T}$ is an $R$-linear map satisfying $\delta([x, y])=$ $[\delta(x), y]+[x, \delta(y)]$ for any $x, y \in \mathscr{T}$ with $x y=0$, then $\delta=d+\tau$, where $d$ is a derivation of $\mathscr{T}$ and $\tau: \mathscr{T} \rightarrow Z(\mathscr{T})$ is an $R$-linear map vanishing at commutators $[x, y]$ with $x y=0$. Bai and $\mathrm{Du}[14]$ proved that letting $\mathscr{M}$ be a von Neumann algebra with no central summands of type $I_{1}$, if $\Phi: \mathscr{M} \rightarrow \mathscr{M}$ is a nonlinear Lie derivation, then $\Phi$ is of the form $\sigma+\tau$, where $\sigma$ is an additive derivation of $\mathscr{M}$ and $\tau$ is a mapping of $\mathscr{M}$ into its center $Z_{\mathscr{M}}$ which maps commutators into zero.

Nevertheless, it would seem desirable to investigate nonlinear Lie derivations of $B(X)$. The present investigation attempts to do this by using the properties of nonlinear Lie derivation and previous research. A map $\delta: B(X) \rightarrow B(X)$ is called an additive derivation if it is additive and satisfies $\delta(A B)=\delta(A) B+A \delta(B)$ for any $A, B \in B(X)$. A linear map $\delta: B(X) \rightarrow B(X)$ is called a Lie derivation if $\delta([A B])=$ $[\delta(A), B]+[A, \delta(B)]$ for any $A, B \in B(X)$, where $[A, B]=$ $A B-B A$ is the usual Lie product. More generally, a map $\Phi: B(X) \rightarrow B(X)$ (without the additive assumption) is a nonlinear Lie derivation if $\Phi([A B])=[\Phi(A), B]+[A, \Phi(B)]$ for any $A, B \in B(X)$.

Recall that $B(X)$ is called prime algebra if $a B(X) b=0$ implies $a=0$ or $=0$. An operator $P \in B(X)$ is an idempotent provided that $P^{2}=P$. In this paper, we suppose that $B(X)$ is a prime associate algebra. We prove that every nonlinear Lie derivation of $B(X)$ is the sum of an additive derivation and a map from $B(X)$ into its center sending commutators to the zero. We want to mention here that there is no additive assumed.

\section{Result and Proof}

Fix a nontrivial idempotent $P_{1} \in B(X)$ and let $P_{2}=I-P_{1}$. In what follows, we write $\mathscr{A}_{i j}=P_{i} B(X) P_{j}$ for, $j=1,2$. Then 
every operator $A \in B(X)$ can be written as $A=\sum_{i, j=1}^{2} A_{i j}$. Note that $A_{i j}$ notation denotes an arbitrary element of $\mathscr{A}_{i j}$.

Definition 1. Letting $S$ be a set of $B(X)$, we call

$$
Z_{S}=\{A \in B(X) \mid[A, B]=0, \forall B \in S\}
$$

the centralizer of $S$ about $B(X)$.

Clearly, $Z_{B(X)}$ is the center of $B(X)$.

Lemma 2. Consider that $Z_{\mathscr{A}_{i j}}=\mathscr{A}_{i j}+Z_{B(X)}, 1 \leq i \neq j \leq 2$.

Proof. It is clearly that $\mathscr{A}_{i j}+Z_{B(X)} \subseteq Z_{\mathscr{A}_{i j}}, 1 \leq i \neq j \leq 2$.

Conversely, without loss of generality, we proof that $Z_{\mathscr{A}_{12}} \subseteq \mathscr{A}_{12}+Z_{B(X)}$. For any $T=\sum_{i, j=1}^{2} T_{i j} \in Z_{\mathscr{A}_{12}}$, we have

$$
T_{11} A_{12}+T_{21} A_{12}=A_{12} T_{22}+A_{12} T_{21}, \quad \forall A_{12} \in \mathscr{A}_{12} .
$$

Thus

$$
\begin{gathered}
T_{21} A_{12}=T_{21} P_{1} A P_{2}=0, \quad \forall A \in B(X), \\
T_{11} A_{12}=A_{12} T_{22}, \quad \forall A_{12} \in \mathscr{A}_{12} .
\end{gathered}
$$

From (3), noting that $B(X)$ is prime, we obtain $T_{21} P_{1}=0$, that is, $T_{21}=0$. By $A_{11} A_{12} \in \mathscr{A}_{12}$, we have

$$
\begin{aligned}
& T_{11} A_{11} A_{12}=A_{11} A_{12} T_{22}=A_{11} T_{11} A_{12}, \\
& \left(T_{11} A_{11}-A_{11} T_{11}\right) P_{1} A P_{2}=0, \quad \forall A \in B(X) .
\end{aligned}
$$

Since $B(X)$ is prime, we have $T_{11} A_{11}=A_{11} T_{11}$. We write $T_{11}=Z_{1} P_{1}$, where $Z_{1} \in Z_{B(X)}$. Since $A_{12} A_{22} \in \mathscr{A}_{12}$, we have

$$
\begin{gathered}
T_{11} A_{12} A_{22}=A_{12} A_{22} T_{22}=A_{12} T_{22} A_{22}, \\
P_{1} A P_{2}\left(A_{22} T_{22}-T_{22} A_{22}\right)=0, \quad \forall A \in B(X) .
\end{gathered}
$$

Since $B(X)$ is prime we have $T_{22} A_{22}=A_{22} T_{22}$. We write $T_{22}=$ $Z_{2} P_{2}$, where $Z_{2} \in Z_{B(X)}$. From (4) we have

$$
\begin{aligned}
\left(Z_{1}-Z_{2}\right) P_{1} A P_{2} & =Z_{1} P_{1} A P_{2}-P_{1} A P_{2} Z_{2} P_{2} \\
& =T_{11} P_{1} A P_{2}-P_{1} A P_{2} T_{22}=0 .
\end{aligned}
$$

So

$$
\left(Z_{1}-Z_{2}\right) P_{1}=0
$$

Thus

$$
\left(Z_{1}-Z_{2}\right) A P_{1}=A\left(Z_{1}-Z_{2}\right) P_{1}=0, \quad \forall A \in B(X) .
$$

So

$$
Z_{1}=Z_{2}
$$

It follows that

$$
\begin{aligned}
T & =T_{12}+Z_{1} P_{1}+Z_{2} P_{2} \\
& =T_{12}+Z_{1} \in \mathscr{A}_{12}+Z_{B(X)} .
\end{aligned}
$$

Lemma 3. Consider that $Z_{B(X)}=Z_{\mathscr{A}_{12}} \cap Z_{\mathscr{A}_{21}}$.

Proof. From Lemma 2, we have

$$
Z_{\mathscr{A}_{12}} \cap Z_{\mathscr{A}_{21}}=\left(\mathscr{A}_{12}+Z_{B(X)}\right) \cap\left(\mathscr{A}_{21}+Z_{B(X)}\right)=Z_{B(X)} .
$$

Theorem 4. Let $X$ be an infinite dimensional Banach space, and $\Phi: B(X) \rightarrow B(X)$ is a nonlinear Lie derivation. Then $\Phi$ is the form $\delta+\tau$, where $\delta$ is an additive derivation of $B(X)$ and $\tau$ is a map from $B(X)$ into its center $Z_{B(X)}$, which maps commutators into the zero.

Proof. Now we organize the proof in a series of claims.

Claim 1. Consider that $\Phi(0)=0$; moreover, $T_{i} \in B(X)$ such that $\Phi\left(P_{i}\right)-\left[P_{i}, T_{i}\right] \in Z_{B(X)}$ and $\Phi\left(A_{i j}\right)=P_{i} \Phi\left(A_{i j}\right) P_{j}+$ $\left[A_{i j}, T_{i}\right], \Phi\left(A_{j i}\right)=P_{j} \Phi\left(A_{j i}\right) P_{i}+\left[A_{j i}, T_{i}\right]$ for arbitrarily $A_{i j} \in$ $\mathscr{A}_{i j}(1 \leq i \neq j \leq 2)$.

Obviously $\Phi(0)=\Phi([0,0])=[\Phi(0), 0]+[0, \Phi(0)]=0$. Moreover, for arbitrarily $A_{i j}$,

$$
\begin{aligned}
\Phi\left(A_{i j}\right)= & \Phi\left(\left[P_{i}, A_{i j}\right]\right)=\left[\Phi\left(P_{i}\right), A_{i j}\right]+\left[P_{i}, \Phi\left(A_{i j}\right)\right] \\
= & \Phi\left(P_{i}\right) A_{i j}-A_{i j} \Phi\left(P_{i}\right)+P_{i} \Phi\left(A_{i j}\right) \\
& -\Phi\left(A_{i j}\right) P_{i} .
\end{aligned}
$$

In (13), the left and right sides are, respectively, multiplied by $P_{i}$ and $P_{j}$, and we have

$$
P_{i} \Phi\left(P_{i}\right) P_{i} A_{i j}=A_{i j} P_{j} \Phi\left(P_{i}\right) P_{j}
$$

In (13), the left and right sides are respectively multiplying by $P_{j}$ and $P_{i}$, and we arrive at

$$
P_{j} \Phi\left(A_{i j}\right) P_{i}=0
$$

For arbitrarily $A_{j i}$

$$
\begin{aligned}
\Phi\left(A_{j i}\right)= & \Phi\left(\left[A_{j i}, P_{i}\right]\right)=\left[\Phi\left(A_{j i}\right), P_{i}\right]+\left[A_{j i}, \Phi\left(P_{i}\right)\right] \\
= & \Phi\left(A_{j i}\right) P_{i}-P_{i} \Phi\left(A_{j i}\right)+A_{j i} \Phi\left(P_{i}\right) \\
& -\Phi\left(P_{i}\right) A_{j i} .
\end{aligned}
$$

In the previous equation, the left and right sides are, respectively multiplied by $P_{j}$ and $P_{i}$, and we have

$$
A_{j i} P_{i} \Phi\left(P_{i}\right) P_{i}=P_{j} \Phi\left(P_{i}\right) P_{j} A_{j i} .
$$

This together with (14) shows that

$$
\begin{aligned}
& {\left[P_{i} \Phi\left(P_{i}\right) P_{i}+P_{j} \Phi\left(P_{i}\right) P_{j}, A_{i j}\right]} \\
& \quad=\left[P_{i} \Phi\left(P_{i}\right) P_{i}+P_{j} \Phi\left(P_{i}\right) P_{j}, A_{j i}\right]=0 .
\end{aligned}
$$


From Lemma 3, it is that

$$
P_{i} \Phi\left(P_{i}\right) P_{i}+P_{j} \Phi\left(P_{i}\right) P_{j} \in Z_{B(X)}
$$

Denote $T_{i}=P_{i} \Phi\left(P_{i}\right) P_{j}-P_{j} \Phi\left(P_{i}\right) P_{i}$. Therefore

$$
\Phi\left(P_{i}\right)-\left[P_{i}, T_{i}\right]=P_{i} \Phi\left(P_{i}\right) P_{i}+P_{j} \Phi\left(P_{i}\right) P_{j} \in Z_{B(X)} .
$$

At the same time, $\left[A_{i j}, T_{i}\right]=P_{j} \Phi\left(P_{i}\right) P_{i} A_{i j}-A_{i j} P_{j} \Phi\left(P_{i}\right) P_{i}$. From (14),

$$
\begin{aligned}
{\left[A_{i j}, T_{i}\right]=} & P_{j} \Phi\left(P_{i}\right) P_{i} A_{i j}-A_{i j} P_{j} \Phi\left(P_{i}\right) P_{i} \\
& +P_{i} \Phi\left(P_{i}\right) P_{i} A_{i j}-A_{i j} P_{j} \Phi\left(P_{i}\right) P_{j} \\
= & \Phi\left(P_{i}\right) A_{i j}-A_{i j} \Phi\left(P_{i}\right) .
\end{aligned}
$$

Because $P_{j} \Phi\left(A_{i j}\right) P_{i}=0$ so by (13) we get $\Phi\left(A_{i j}\right)=$ $P_{i} \Phi\left(A_{i j}\right) P_{j}+\left[A_{i j}, T_{i}\right]$. $\left[A_{j i}, T_{i}\right]$.

In the same way, we can get $\Phi\left(A_{j i}\right)=P_{j} \Phi\left(A_{j i}\right) P_{i}+$

Remark. It is clear that $T \rightarrow\left[T, T_{i}\right]$ is an additive derivation. Without loss of generality, we can assume that $\Phi\left(P_{1}\right) \in Z_{B(X)}$ and $\Phi\left(\mathscr{A}_{i j}\right) \subseteq \mathscr{A}_{i j}(1 \leq i \neq j \leq 2)$.

Claim 2. Consider that $\Phi\left(\mathscr{A}_{i i}\right) \subseteq \mathscr{A}_{i i}+Z_{B(X)}(i=1,2)$.

Let $i=1$, for every $T_{11} \in \mathscr{A}_{11}$, and write $\Phi\left(T_{11}\right)=$ $\sum_{i, j=1}^{2} B_{i j}$. From $\Phi\left(P_{1}\right) \in Z_{B(X)}$ and $\left[P_{1}, A\right]=\left[A, P_{2}\right]$, we can get $\Phi\left(P_{2}\right) \in Z_{B(X)}$ :

$$
\begin{aligned}
0 & =\Phi\left(\left[T_{11}, P_{2}\right]\right)=\left[\Phi\left(T_{11}\right), P_{2}\right]+\left[T_{11}, \Phi\left(P_{2}\right)\right] \\
& =\Phi\left(T_{11}\right) P_{2}-P_{2} \Phi\left(T_{11}\right) .
\end{aligned}
$$

From this $P_{1} \Phi\left(T_{11}\right) P_{2}=P_{2} \Phi\left(T_{11}\right) P_{1}=0$. So we have $B_{12}=$ $B_{21}=0$. For every $A_{22} \in \mathscr{A}_{22}$,

$$
\begin{aligned}
0 & =\Phi\left(\left[T_{11}, A_{22}\right]\right)=\left[\Phi\left(T_{11}\right), A_{22}\right]+\left[T_{11}, \Phi\left(A_{22}\right)\right] \\
& =B_{22} A_{22}-A_{22} B_{22}+T_{11} \Phi\left(A_{22}\right)-\Phi\left(A_{22}\right) T_{11}
\end{aligned}
$$

Thus, $B_{22} A_{22}-A_{22} B_{22}=0$, and so $B_{22}=P_{2} Z$ for some $\epsilon$ $Z_{B(X)}$. Hence

$$
\Phi\left(T_{11}\right)=B_{11}-P_{1} Z+Z \in \mathscr{A}_{11}+Z_{B(X)} .
$$

The other case can be treated similarly.

Claim 3. $\Phi$ is almost additive map; that is, for every T, $S \in$ $B(X), \Phi(T+S)-\Phi(T)-\Phi(S) \in Z_{B(X)}$.

We divided the proof into several steps.

Step 1. For every $T_{i i}, T_{j i}$, and $T_{i j}(1 \leq i \neq j \leq 2), \Phi\left(T_{i i}+T_{i j}\right)-$ $\Phi\left(T_{i i}\right)-\Phi\left(T_{i j}\right) \in Z_{B(X)}, \Phi\left(T_{i i}+T_{j i}\right)-\Phi\left(T_{i i}\right)-\Phi\left(T_{j i}\right) \in Z_{B(X)}$.
Let $=1$, and let $j=2$. For every $A_{12} \in \mathscr{A}_{12},\left[T_{11}+\right.$ $\left.T_{12}, A_{12}\right]=\left[T_{11}, A_{12}\right]$; therefore

$$
\begin{gathered}
{\left[\Phi\left(T_{11}+T_{12}\right), A_{12}\right]+\left[T_{11}+T_{12}, \Phi\left(A_{12}\right)\right]} \\
\quad=\left[\Phi\left(T_{11}\right), A_{12}\right]+\left[T_{11}, \Phi\left(A_{12}\right)\right] .
\end{gathered}
$$

This yields $\left[\Phi\left(T_{11}+T_{12}\right)-\Phi\left(T_{11}\right), A_{12}\right]=0$. From Lemma 2 ,

$$
\begin{aligned}
& \Phi\left(T_{11}+T_{12}\right)-\Phi\left(T_{11}\right) \\
& \quad=P_{1}\left(\Phi\left(T_{11}+T_{12}\right)-\Phi\left(T_{11}\right)\right) P_{2}+Z
\end{aligned}
$$

for $\in Z_{B(X)}$.

Since $\Phi\left(P_{1}\right) \in Z_{B(X)}$ and $\left[P_{1}, T\right]=P_{1} T P_{2}$ for all $T \in \mathscr{A}_{11} \oplus$ $\mathscr{A}_{12} \oplus \mathscr{A}_{22}$. We have that

$$
\begin{aligned}
\Phi\left(P_{1} T P_{2}\right) & =\Phi\left(\left[P_{1}, T\right]\right) \\
& =\left[\Phi\left(P_{1}\right), T\right]+\left[P_{1}, \Phi(T)\right]=P_{1} \Phi(T) P_{2} .
\end{aligned}
$$

Then

$$
\begin{aligned}
P_{1} & \left(\Phi\left(T_{11}+T_{12}\right)-\Phi\left(T_{11}\right)\right) P_{2} \\
& =\Phi\left(P_{1}\left(T_{11}+T_{12}\right) P_{2}\right)-\Phi\left(P_{1}\left(T_{11}\right) P_{2}\right) \\
& =\Phi\left(P_{1} T_{11} P_{2}+P_{1} T_{12} P_{2}\right)-\Phi\left(P_{1} T_{11} P_{2}\right) \\
& =\Phi\left(P_{1} T_{12} P_{2}\right)=\Phi\left(T_{12}\right) .
\end{aligned}
$$

It shows that $\Phi\left(T_{11}+T_{12}\right)-\Phi\left(T_{11}\right)=\Phi\left(T_{12}\right)+Z$. The rest of the other hand is also the same reason.

Step 2. $\Phi$ is additive on $\mathscr{A}_{12}$ and $\mathscr{A}_{21}$.

For $T_{12}, S_{12} \in \mathscr{A}_{12}$. Because $T_{12}+S_{12}=\left[P_{1}+T_{12}, P_{2}+S_{12}\right]$, by Step 1, we know that

$$
\begin{aligned}
\Phi\left(T_{12}+S_{12}\right)= & {\left[\Phi\left(P_{1}+T_{12}\right), P_{2}+S_{12}\right] } \\
& +\left[P_{1}+T_{12}, \Phi\left(P_{2}+S_{12}\right)\right] \\
= & {\left[\Phi\left(P_{1}\right)+\Phi\left(T_{12}\right), P_{2}+S_{12}\right] } \\
& +\left[P_{1}+T_{12}, \Phi\left(P_{2}\right)+\Phi\left(S_{12}\right)\right] \\
= & \Phi\left(T_{12}\right)+\Phi\left(S_{12}\right) .
\end{aligned}
$$

The rest is similar.

Step 3. For any $T_{11} \in \mathscr{A}_{11}, T_{22} \in \mathscr{A}_{22}, \Phi\left(T_{11}+T_{22}\right)-\Phi\left(T_{11}\right)-$ $\Phi\left(T_{22}\right) \in Z_{B(X)}$.

Let $A_{12} \in \mathscr{A}_{12}$, and $\left[T_{11}+T_{22}, A_{12}\right]=T_{11} A_{12}-A_{12} T_{22}$. For Step 2, we know that

$$
\begin{aligned}
{[\Phi} & \left.\left(T_{11}+T_{22}\right), A_{12}\right]+\left[T_{11}+T_{22}, \Phi\left(A_{12}\right)\right] \\
= & \Phi\left(T_{11} A_{12}\right)+\Phi\left(-A_{12} T_{22}\right) \\
= & \Phi\left(\left[T_{11}, A_{12}\right]\right)+\Phi\left(\left[T_{22}, A_{12}\right]\right) \\
= & {\left[\Phi\left(T_{11}\right), A_{12}\right]+\left[T_{11}, \Phi\left(A_{12}\right)\right] } \\
& +\left[\Phi\left(T_{22}\right), A_{12}\right]+\left[T_{22}, \Phi\left(A_{12}\right)\right] .
\end{aligned}
$$


So $\left[\Phi\left(T_{11}+T_{22}\right)-\Phi\left(T_{11}\right)-\Phi\left(T_{22}\right), A_{12}\right]=0$. From Lemma 2 ,

$$
\Phi\left(T_{11}+T_{22}\right)-\Phi\left(T_{11}\right)-\Phi\left(T_{22}\right) \in \mathscr{A}_{12}+Z_{B(X)}
$$

On the other hand, from (27) we get that $P_{1}\left(\Phi\left(T_{11}+T_{22}\right)-\right.$ $\left.\Phi\left(T_{11}\right)-\Phi\left(T_{22}\right)\right) P_{2}=0$. So

$$
\Phi\left(T_{11}+T_{22}\right)-\Phi\left(T_{11}\right)-\Phi\left(T_{22}\right) \in Z_{B(X)}
$$

Step 4. For any $T_{i i}, S_{i i} \in \mathscr{A}_{i i}(i=1,2), \Phi\left(T_{i i}+S_{i i}\right)-\Phi\left(T_{i i}\right)-$ $\Phi\left(S_{i i}\right) \in Z_{B(X)}$.

Let $i=1$. For any $A_{12} \in \mathscr{A}_{12},\left[T_{11}+S_{11}, A_{12}\right]=T_{11} A_{12}+$ $S_{11} A_{12}$. From Step 2, it is that

$$
\begin{aligned}
& {\left[\Phi\left(T_{11}+S_{11}\right), A_{12}\right]+\left[T_{11}+S_{11}, \Phi\left(A_{12}\right)\right]} \\
& =\Phi\left(T_{11} A_{12}\right)+\Phi\left(S_{11} A_{12}\right) \\
& =\Phi\left(\left[T_{11}, A_{12}\right]\right)+\Phi\left(\left[S_{11}, A_{12}\right]\right) \\
& =\left[\Phi\left(T_{11}\right), A_{12}\right]+\left[T_{11}, \Phi\left(A_{12}\right)\right] \\
& \quad+\left[\Phi\left(S_{11}\right), A_{12}\right]+\left[S_{11}, \Phi\left(A_{12}\right)\right] .
\end{aligned}
$$

So $\left[\Phi\left(T_{11}+S_{11}\right)-\Phi\left(T_{11}\right)-\Phi\left(S_{11}\right), A_{12}\right]=0$. From Lemma 2 ,

$$
\Phi\left(T_{11}+S_{11}\right)-\Phi\left(T_{11}\right)-\Phi\left(S_{11}\right) \in \mathscr{A}_{12}+Z_{B(X)}
$$

On the other hand, from (27) we get that $P_{1}\left(\Phi\left(T_{11}+S_{11}\right)-\right.$ $\left.\Phi\left(T_{11}\right)-\Phi\left(S_{11}\right)\right) P_{2}=0$. So

$$
\Phi\left(T_{11}+S_{11}\right)-\Phi\left(T_{11}\right)-\Phi\left(S_{11}\right) \in Z_{B(X)}
$$

The other case can be treated similarly.

Step 5. Consider that $\Phi\left(T_{i i}+T_{j j}+T_{i j}\right)-\Phi\left(T_{i i}\right)-\Phi\left(T_{j j}\right)-$ $\Phi\left(T_{i j}\right) \in Z_{B(X)}$.

Let $i=1$, and let $j=2$. For any $A_{12} \in \mathscr{A}_{12},\left[T_{11}+T_{22}+\right.$ $\left.T_{12}, A_{12}\right]=\left[T_{11}+T_{22}, A_{12}\right]$. By Step 3 , it is that

$$
\begin{array}{r}
{\left[\Phi\left(T_{11}+T_{22}+T_{12}\right), A_{12}\right]+\left[T_{11}+T_{22}+T_{12}, \Phi\left(A_{12}\right)\right]} \\
=\left[\Phi\left(T_{11}\right)+\Phi\left(T_{22}\right), A_{12}\right]+\left[T_{11}+T_{22}, \Phi\left(A_{12}\right)\right] .
\end{array}
$$

So $\left[\Phi\left(T_{11}+T_{22}+T_{12}\right)-\Phi\left(T_{11}\right)-\Phi\left(T_{22}\right), A_{12}\right]=0$. From Lemma 2, it follows that

$$
\begin{aligned}
& \Phi\left(T_{11}+T_{22}+T_{12}\right)-\Phi\left(T_{11}\right)-\Phi\left(T_{22}\right) \\
& \quad=P_{1}\left(\Phi\left(T_{11}+T_{22}+T_{12}\right)-\Phi\left(T_{11}\right)-\Phi\left(T_{22}\right)\right) P_{2}+Z
\end{aligned}
$$

for some central element $Z \in Z_{B(X)}$. From (27) we get that

$$
\Phi\left(T_{12}\right)=P_{1} \Phi\left(T_{11}+T_{22}+T_{12}\right) P_{2}
$$

So

$$
\Phi\left(T_{11}+T_{22}+T_{12}\right)-\Phi\left(T_{11}\right)-\Phi\left(T_{22}\right)-\Phi\left(T_{12}\right) \in Z_{B(X)} .
$$

Similarly, $\Phi\left(T_{22}+T_{11}+T_{21}\right)-\Phi\left(T_{22}\right)-\Phi\left(T_{11}\right)-\Phi\left(T_{21}\right) \in Z_{B(X)}$. Step 6. For all $T, S \in B(X), \Phi(T+S)-\Phi(T)-\Phi(S) \in Z_{B(X)}$. Let $T \in B(X)$, and write $T=T_{11}+T_{12}+T_{21}+T_{22}$. If we know that

$$
\begin{aligned}
& \Phi\left(T_{11}+T_{12}+T_{21}+T_{22}\right)-\Phi\left(T_{11}\right) \\
& \quad-\Phi\left(T_{12}\right)-\Phi\left(T_{21}\right)-\Phi\left(T_{22}\right) \in Z_{B(X)},
\end{aligned}
$$

then it is right.

For every $A_{12} \in \mathscr{A}_{12},\left[T_{11}+T_{12}+T_{21}+T_{22}, A_{12}\right]=\left[T_{11}+\right.$ $\left.T_{21}+T_{22}, A_{12}\right]$. By Step 5, it shows that

$$
\begin{aligned}
& {\left[\Phi\left(T_{11}+T_{12}+T_{21}+T_{22}\right), A_{12}\right]} \\
& \quad+\left[T_{11}+T_{12}+T_{21}+T_{22}, \Phi\left(A_{12}\right)\right] \\
& =\left[\Phi\left(T_{11}\right)+\Phi\left(T_{21}\right)+\Phi\left(T_{22}\right), A_{12}\right] \\
& \quad+\left[T_{11}+T_{21}+T_{22}, \Phi\left(A_{12}\right)\right] .
\end{aligned}
$$

So

$$
\begin{aligned}
& {\left[\Phi\left(T_{11}+T_{12}+T_{21}+T_{22}\right)\right.} \\
& \left.\quad-\Phi\left(T_{11}\right)-\Phi\left(T_{21}\right)-\Phi\left(T_{22}\right), A_{12}\right]=0 .
\end{aligned}
$$

Because $\Phi\left(T_{12}\right) \in \mathscr{A}_{12}$, we clearly have

$$
\begin{aligned}
& {\left[\Phi\left(T_{11}+T_{12}+T_{21}+T_{22}\right)-\Phi\left(T_{11}\right)\right.} \\
& \left.\quad-\Phi\left(T_{12}\right)-\Phi\left(T_{21}\right)-\Phi\left(T_{22}\right), A_{12}\right]=0 .
\end{aligned}
$$

Similarly, $\left[T_{11}+T_{12}+T_{21}+T_{22}, A_{21}\right]=\left[T_{11}+T_{12}+T_{22}, A_{21}\right]$, and we can obtain

$$
\begin{aligned}
& {\left[\Phi\left(T_{11}+T_{12}+T_{21}+T_{22}\right)-\Phi\left(T_{11}\right)\right.} \\
& \left.\quad-\Phi\left(T_{12}\right)-\Phi\left(T_{21}\right)-\Phi\left(T_{22}\right), A_{21}\right]=0 .
\end{aligned}
$$

From Lemma 3,

$$
\begin{aligned}
& \Phi\left(T_{11}+T_{12}+T_{21}+T_{22}\right)-\Phi\left(T_{11}\right) \\
& \quad-\Phi\left(T_{12}\right)-\Phi\left(T_{21}\right)-\Phi\left(T_{22}\right) \in Z_{B(X)}
\end{aligned}
$$

For every $A \in B(X)$, from Step 6, we define a mapping $\tau$ of $B(X)$ into $Z_{B(X)}$ by

$$
\begin{aligned}
\tau(A)= & \Phi(A)-\Phi\left(P_{1} A P_{1}\right) \\
& -\Phi\left(P_{1} A P_{2}\right)-\Phi\left(P_{2} A P_{1}\right)-\Phi\left(P_{2} A P_{2}\right) .
\end{aligned}
$$

Then let $\delta(A)=\Phi(A)-\tau(A)$.

In the following, we will prove that $\delta$ and $\tau$ are desired maps. 
Claim $4 . \delta$ is an additive derivation.

We divide the proof into the following steps.

Step $1 . \delta$ is an additive map.

We only need to show that $\delta$ is an additive on $\mathscr{A}_{i i}$. Let $A_{i i}, A_{i i}^{\prime} \in \mathscr{A}_{i i}$,

$$
\begin{aligned}
\delta\left(A_{i i}+A_{i i}^{\prime}\right)-\delta\left(A_{i i}\right)-\delta\left(A_{i i}^{\prime}\right) \\
=\Phi\left(A_{i i}+A_{i i}^{\prime}\right)-\tau\left(A_{i i}+A_{i i}^{\prime}\right)-\Phi\left(A_{i i}\right) \\
\quad+\tau\left(A_{i i}\right)-\Phi\left(A_{i i}^{\prime}\right)+\tau\left(A_{i i}^{\prime}\right) .
\end{aligned}
$$

Thus

$$
\delta\left(A_{i i}+A_{i i}^{\prime}\right)-\delta\left(A_{i i}\right)-\delta\left(A_{i i}^{\prime}\right) \in Z_{B(X)} \cap \mathscr{A}_{i i}=\{0\},
$$

and so $\delta\left(A_{i i}+A_{i i}^{\prime}\right)=\delta\left(A_{i i}\right)+\delta\left(A_{i i}^{\prime}\right)$.

Step 2. For every $T_{i i}, S_{i i} \in \mathscr{A}_{i i}, T_{i j}, S_{i j} \in \mathscr{A}_{i j}, S_{j j} \in \mathscr{A}_{j j}(1 \leq$ $i \neq j \leq 2)$, and $\delta\left(T_{i i} S_{i j}\right)=\delta\left(T_{i i}\right) S_{i j}+T_{i i} \delta\left(S_{i j}\right), \delta\left(T_{i j} S_{j j}\right)=$ $\delta\left(T_{i j}\right) S_{j j}+T_{i j} \delta\left(S_{j j}\right), \delta\left(T_{i i} S_{i i}\right)=\delta\left(T_{i i}\right) S_{i i}+T_{i i} \delta\left(S_{i i}\right)$.

Let $T_{i j}, S_{i j}, A_{i j} \in \mathscr{A}_{i j}(i \neq j)$,

$$
\begin{aligned}
\delta\left(T_{i i} S_{i j}\right) & =\Phi\left(T_{i i} S_{i j}\right)=\Phi\left(\left[T_{i i}, S_{i j}\right]\right) \\
& =\left[\Phi\left(T_{i i}\right), S_{i j}\right]+\left[T_{i i}, \Phi\left(S_{i j}\right)\right] \\
& =\left[\delta\left(T_{i i}\right), S_{i j}\right]+\left[T_{i i}, \delta\left(S_{i j}\right)\right] \\
& =\delta\left(T_{i i}\right) S_{i j}+T_{i i} \delta\left(S_{i j}\right), \\
\delta\left(T_{i j} S_{j j}\right) & =\Phi\left(T_{i j} S_{j j}\right)=\Phi\left(\left[T_{i j}, S_{j j}\right]\right) \\
& =\left[\Phi\left(T_{i j}\right), S_{j j}\right]+\left[T_{i j}, \Phi\left(S_{j j}\right)\right] \\
& =\left[\delta\left(T_{i j}\right) S_{j j}\right]+\left[T_{i j} \delta\left(S_{j j}\right)\right] \\
& =\delta\left(T_{i j}\right) S_{j j}+T_{i j} \delta\left(S_{j j}\right) .
\end{aligned}
$$

So

$$
\delta\left(T_{i i} S_{i i} A_{i j}\right)=\delta\left(T_{i i} S_{i i}\right) A_{i j}+T_{i i} S_{i i} \delta\left(A_{i j}\right) .
$$

On the other hand,

$$
\begin{aligned}
\delta\left(T_{i i} S_{i i} A_{i j}\right) & =\delta\left(T_{i i}\right) S_{i i} A_{i j}+T_{i i} \delta\left(S_{i i} A_{i j}\right) \\
& =\delta\left(T_{i i}\right) S_{i i} A_{i j}+T_{i i} \delta\left(S_{i i}\right) A_{i j}+T_{i i} S_{i i} \delta\left(A_{i j}\right) .
\end{aligned}
$$

So $\left(\delta\left(T_{i i} S_{i i}\right)-\delta\left(T_{i i}\right) S_{i i}-T_{i i} \delta\left(S_{i i}\right)\right) A_{i j}=0$. Note that $B(X)$ is prime, so

$$
\delta\left(T_{i i} S_{i i}\right)=\delta\left(T_{i i}\right) S_{i i}+T_{i i} \delta\left(S_{i i}\right)
$$

Step 3. $\delta$ is an additive derivation on $\mathscr{A}_{11} \oplus \mathscr{A}_{12} \oplus \mathscr{A}_{22}$ and $\mathscr{A}_{11} \oplus \mathscr{A}_{21} \oplus \mathscr{A}_{22}$.
Let $A=A_{11}+A_{12}+A_{22}$ and let $B=B_{11}+B_{12}+B_{22}$ be in $\mathscr{A}_{11} \oplus \mathscr{A}_{12} \oplus \mathscr{A}_{22}$. By Steps 1 and 2, we have

$$
\begin{aligned}
& \delta(A B)=\delta\left[\left(A_{11}+A_{12}+A_{22}\right)\left(B_{11}+B_{12}+B_{22}\right)\right] \\
& =\delta\left(A_{11} B_{11}\right)+\delta\left(A_{11} B_{12}\right)+\delta\left(A_{12} B_{22}\right)+\delta\left(A_{22} B_{22}\right) \\
& =\delta\left(A_{11}\right) B_{11}+A_{11} \delta\left(B_{11}\right)+\delta\left(A_{11}\right) B_{12}+A_{11} \delta\left(B_{12}\right) \\
& \quad+\delta\left(A_{12}\right) B_{22}+A_{12} \delta\left(B_{22}\right)+\delta\left(A_{22}\right) B_{22}+A_{22} \delta\left(B_{22}\right) .
\end{aligned}
$$

From $\delta\left(\mathscr{A}_{i j}\right) \subseteq \mathscr{A}_{i j}$, we have

$$
\begin{aligned}
& \delta(A) B+A \delta(B) \\
&=\delta\left(A_{11}+A_{12}+A_{22}\right)\left(B_{11}+B_{12}+B_{22}\right) \\
&+\left(A_{11}+A_{12}+A_{22}\right) \delta\left(B_{11}+B_{12}+B_{22}\right) \\
&= \delta\left(A_{11}\right) B_{11}+A_{11} \delta\left(B_{11}\right)+\delta\left(A_{11}\right) B_{12}+A_{11} \delta\left(B_{12}\right) \\
&+\delta\left(A_{12}\right) B_{22}+A_{12} \delta\left(B_{22}\right)+\delta\left(A_{22}\right) B_{22}+A_{22} \delta\left(B_{22}\right) .
\end{aligned}
$$

So $\delta(A) B+A \delta(B)=\delta(A B)$, and so $\delta$ is an additive derivation on $\mathscr{A}_{11} \oplus \mathscr{A}_{12} \oplus \mathscr{A}_{22}$. So from [15, Theorem 4.1] we can see that $\delta$ is a derivation. Furthermore, due to the main result in [6], there exists an operator $T^{\prime} \in B(X)$ such that

$$
\delta(A)=T^{\prime} A-A T^{\prime}
$$

for all $A \in \mathscr{A}_{11} \oplus \mathscr{A}_{12} \oplus \mathscr{A}_{22}$.

Similarly, we can know that $\delta$ is an additive derivation on $\mathscr{A}_{11} \oplus \mathscr{A}_{21} \oplus \mathscr{A}_{22}$, so there exists an operator $S^{\prime} \in B(X)$ such that

$$
\delta(A)=S^{\prime} A-A S^{\prime}
$$

for all $A \in \mathscr{A}_{11} \oplus \mathscr{A}_{21} \oplus \mathscr{A}_{22}$.

Step 4. Consider that $\delta\left(T_{i j} S_{j i}\right)=\delta\left(T_{i j}\right) S_{j i}+T_{i j} \delta\left(S_{j i}\right)$, for every $T_{i j} \in \mathscr{A}_{i j}, S_{j i} \in \mathscr{A}_{j i}(1 \leq i \neq j \leq 2)$.

Now, we write $T^{\prime}=\sum_{i, j=1}^{2} T_{i j}^{\prime}$ and $S^{\prime}=\sum_{i, j=1}^{2} S_{i j}^{\prime}$. Then by Step 3, we have that

$$
\begin{aligned}
& T^{\prime}\left(A_{11}+B_{22}\right)-\left(A_{11}+B_{22}\right) T^{\prime} \\
& \quad=S^{\prime}\left(A_{11}+B_{22}\right)-\left(A_{11}+B_{22}\right) S^{\prime} .
\end{aligned}
$$

For all $A_{11} \in \mathscr{A}_{11}$, for all $B_{22} \in \mathscr{A}_{22}$, this shows that

$$
\begin{aligned}
& \left(T^{\prime}-S^{\prime}\right) A_{11}=A_{11}\left(T^{\prime}-S^{\prime}\right), \\
& \left(T^{\prime}-S^{\prime}\right) B_{22}=B_{22}\left(T^{\prime}-S^{\prime}\right) .
\end{aligned}
$$

Multiplying the previous two equations by $P_{2}$ and $P_{1}$ from the left respectively, we can know that

$$
\left(T_{21}^{\prime}-S_{21}^{\prime}\right) A_{11}=0, \quad\left(T_{12}^{\prime}-S_{12}^{\prime}\right) B_{22}=0 .
$$


Similarly,

$$
\begin{aligned}
& \left(T_{11}^{\prime}-S_{11}^{\prime}\right) A_{11}=A_{11}\left(T_{11}^{\prime}-S_{11}^{\prime}\right), \\
& \left(T_{22}^{\prime}-S_{22}^{\prime}\right) B_{22}=B_{22}\left(T_{22}^{\prime}-S_{22}^{\prime}\right) .
\end{aligned}
$$

So

$$
\begin{array}{cl}
T_{12}^{\prime}=S_{12}^{\prime}, & T_{21}^{\prime}=S_{21}^{\prime}, \\
T_{11}^{\prime}-S_{11}^{\prime}=\lambda_{1} P_{1}, & T_{22}^{\prime}-S_{22}^{\prime}=\lambda_{2} P_{2}
\end{array}
$$

for some $\lambda_{1}, \lambda_{2} \in \mathbb{C}$.

For every $A_{12} \in \mathscr{A}_{12}$ and $B_{21} \in \mathscr{A}_{21}$, using (55), (56), and (61), we get that

$$
\begin{aligned}
\delta & \left(A_{12} B_{21}\right)-\delta\left(A_{12}\right) B_{21}-A_{12} \delta\left(B_{21}\right) \\
= & T^{\prime} A_{12} B_{21}-A_{12} B_{21} T^{\prime}-\left(T^{\prime} A_{12}-A_{12} T^{\prime}\right) B_{21} \\
& -A_{12}\left(S^{\prime} B_{21}-B_{21} S^{\prime}\right) \\
= & -A_{12} B_{21} T^{\prime}+A_{12} T^{\prime} B_{21}-A_{12} S^{\prime} B_{21}+A_{12} B_{21} S^{\prime} \\
= & \left(\lambda_{2}-\lambda_{1}\right) A_{12} B_{21} . \\
\delta & \left(B_{21} A_{12}\right)-\delta\left(B_{21}\right) A_{12}-B_{21} \delta\left(A_{12}\right) \\
= & S^{\prime} B_{21} A_{12}-B_{21} A_{12} S^{\prime}-\left(S^{\prime} B_{21}-B_{21} S^{\prime}\right) A_{12} \\
& -B_{21}\left(S^{\prime} A_{12}-A_{12} S^{\prime}\right) \\
= & -B_{21} A_{12} S^{\prime}+B_{21} S^{\prime} A_{12}-B_{21} T^{\prime} A_{12}+B_{21} A_{12} T^{\prime} \\
= & \left(\lambda_{2}-\lambda_{1}\right) B_{21} A_{12} .
\end{aligned}
$$

Since at least one of $P_{2}$ or $P_{1}$ is of rank greater than $2, A_{12}^{\prime} \in$ $\mathscr{A}_{12}$ and $B_{21}^{\prime} \in \mathscr{A}_{21}$ exist such that

$$
A_{12}^{\prime} B_{21}^{\prime}=0, \quad B_{21}^{\prime} A_{12}^{\prime} \neq 0
$$

or

$$
B_{21}^{\prime} A_{12}^{\prime}=0, \quad A_{12}^{\prime} B_{21}^{\prime} \neq 0 .
$$

We suppose that $A_{12}^{\prime} B_{21}^{\prime}=0$ and $B_{21}^{\prime} A_{12}^{\prime} \neq 0$. From (62), we get

$$
\begin{aligned}
\tau & \left(\left[A_{12}^{\prime}, B_{21}^{\prime}\right]\right)=\Phi\left(\left[A_{12}^{\prime}, B_{21}^{\prime}\right]\right)-\delta\left(\left[A_{12}^{\prime}, B_{21}^{\prime}\right]\right) \\
= & {\left[\Phi\left(A_{12}^{\prime}\right), B_{21}^{\prime}\right]+\left[A_{12}^{\prime}, \Phi\left(B_{21}^{\prime}\right)\right]-\delta\left(\left[A_{12}^{\prime}, B_{21}^{\prime}\right]\right) } \\
= & {\left[\delta\left(A_{12}^{\prime}\right), B_{21}^{\prime}\right]+\left[A_{12}^{\prime}, \delta\left(B_{21}^{\prime}\right)\right]-\delta\left(\left[A_{12}^{\prime}, B_{21}^{\prime}\right]\right) } \\
= & \delta\left(A_{12}^{\prime}\right) B_{21}^{\prime}-B_{21}^{\prime} \delta\left(A_{12}^{\prime}\right)+A_{12}^{\prime} \delta\left(B_{21}^{\prime}\right) \\
& -\delta\left(B_{21}^{\prime}\right) A_{12}^{\prime}-\delta\left(A_{12}^{\prime} B_{21}^{\prime}\right)+\delta\left(B_{21}^{\prime} A_{12}^{\prime}\right) \\
= & \left(\lambda_{1}-\lambda_{2}\right) A_{12}^{\prime} B_{21}^{\prime}+\left(\lambda_{2}-\lambda_{1}\right) B_{21}^{\prime} A_{12}^{\prime} \\
= & \left(\lambda_{2}-\lambda_{1}\right) B_{21}^{\prime} A_{12}^{\prime} .
\end{aligned}
$$

From $\tau\left(\left[A_{12}^{\prime}, B_{21}^{\prime}\right]\right) \in Z_{B(X)}$ and $B_{21}^{\prime} A_{12}^{\prime} \neq 0$, then $\lambda_{1}=\lambda_{2}$. So

$$
\begin{gathered}
\delta\left(A_{12} B_{21}\right)=\delta\left(A_{12}\right) B_{21}+A_{12} \delta\left(B_{21}\right), \\
\delta\left(B_{21} A_{12}\right)=\delta\left(B_{21}\right) A_{12}+B_{21} \delta\left(A_{12}\right)
\end{gathered}
$$

for any $A_{12} \in \mathscr{A}_{12}$ and $B_{21} \in \mathscr{A}_{21}$. So

$$
\begin{aligned}
& \delta\left(T_{i j} S_{j i}\right)=\delta\left(T_{i j}\right) S_{j i}+T_{i j} \delta\left(S_{j i}\right), \\
& \delta\left(S_{j i} T_{i j}\right)=\delta\left(S_{j i}\right) T_{i j}+S_{j i} \delta\left(T_{i j}\right) .
\end{aligned}
$$
so

Let $T, S \in B(X)$, and write $T=\sum_{i, j=1}^{2} T_{i j}$ and $S=\sum_{i, j=1}^{2} S_{i j}$,

$$
\begin{aligned}
\delta(T S)= & \delta\left(T_{11} S_{11}\right)+\delta\left(T_{11} S_{12}\right)+\delta\left(T_{12} S_{22}\right)+\delta\left(T_{12} S_{21}\right) \\
& +\delta\left(T_{21} S_{12}\right)+\delta\left(T_{21} S_{11}\right)+\delta\left(T_{22} S_{21}\right)+\delta\left(T_{22} S_{22}\right) \\
= & \delta(T) S+T \delta(S) .
\end{aligned}
$$

Claim 7. $\tau$ sends the commutators into zero:

$$
\begin{aligned}
\tau([A, B])= & \Phi([A, B])-\delta([A, B]) \\
& =[\Phi(A), B]+[A, \Phi(B)]-\delta([A, B]) \\
& =[\delta(A), B]+[A, \delta(B)]-\delta([A, B]) \\
& =0 .
\end{aligned}
$$

The proof is complete.

\section{Acknowledgments}

The authors would like to thank his tutor Professor Baodong Zheng for his valuable suggestions and encouragement. This work was supported by Mudanjiang city science and technology plan Projects (G2013n1446) and Mudanjiang Normal Colllege Youth academic backbone Projects (G201209).

\section{References}

[1] J. Alaminos, M. Mathieu, and A. R. Villena, "Symmetric amenability and Lie derivations," Mathematical Proceedings of the Cambridge Philosophical Society, vol. 137, no. 2, pp. 433-439, 2004.

[2] B. E. Johnson, "Symmetric amenability and the nonexistence of Lie and Jordan derivations," Mathematical Proceedings of the Cambridge Philosophical Society, vol. 120, no. 3, pp. 455-473, 1996.

[3] F. Y. Lu, "Lie derivations of $I$-subspace lattice algebras," Proceedings of the American Mathematical Society, vol. 135, no. 8, pp. 2581-2590, 2007.

[4] F. Y. Lu, "Lie derivations of certain CSL algebras," Israel Journal of Mathematics, vol. 155, pp. 149-156, 2006.

[5] M. Mathieu and A. R. Villena, "The structure of Lie derivations on $C^{*}$-algebras," Journal of Functional Analysis, vol. 202, no. 2, pp. 504-525, 2003.

[6] P. Šemrl, "Additive derivations of some operator algebras," Illinois Journal of Mathematics, vol. 35, no. 2, pp. 234-240, 1991. 
[7] M. I. Berenguer and A. R. Villena, "Continuity of Lie derivations on Banach algebras," Proceedings of the Edinburgh Mathematical Society. Series II, vol. 41, no. 3, pp. 625-630, 1998.

[8] D. Benkovič, "Lie derivations on triangular matrices," Linear and Multilinear Algebra, vol. 55, no. 6, pp. 619-626, 2007.

[9] W.-S. Cheung, "Lie derivations of triangular algebras," Linear and Multilinear Algebra, vol. 51, no. 3, pp. 299-310, 2003.

[10] A. R. Villena, "Lie derivations on Banach algebras," Journal of Algebra, vol. 226, no. 1, pp. 390-409, 2000.

[11] F. Y. Lu and W. Jing, "Characterizations of Lie derivations of $B(X)$," Linear Algebra and its Applications, vol. 432, no. 1, pp. 89-99, 2010.

[12] W. Y. Yu and J.H. Zhang, "Nonlinear Lie derivations of triangular algebras," Linear Algebra and its Applications, vol. 432, no. 11, pp. 2953-2960, 2010.

[13] P. S. Ji and W. Q. Qi, "Characterizations of Lie derivations of triangular algebras," Linear Algebra and its Applications, vol. 435, no. 5, pp. 1137-1146, 2011.

[14] Z. F. Bai and S. P. Du, "The structure of nonlinear Lie derivation on von Neumann algebras," Linear Algebra and its Applications, vol. 436, no. 7, pp. 2701-2708, 2012.

[15] D. G. Han, "Continuity and linearity of additive derivations of nest algebras on Banach spaces," Chinese Annals of Mathematics $B$, vol. 17, no. 2, pp. 227-236, 1996. 


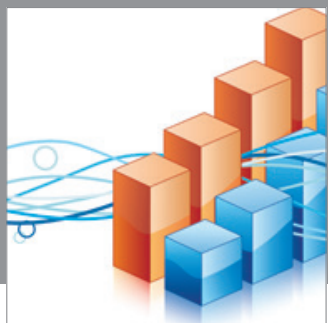

Advances in

Operations Research

mansans

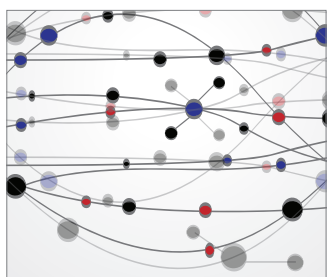

The Scientific World Journal
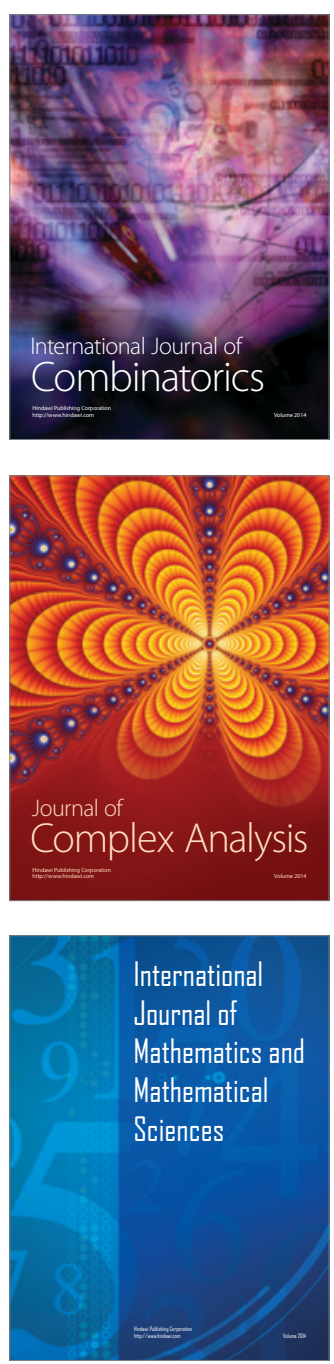
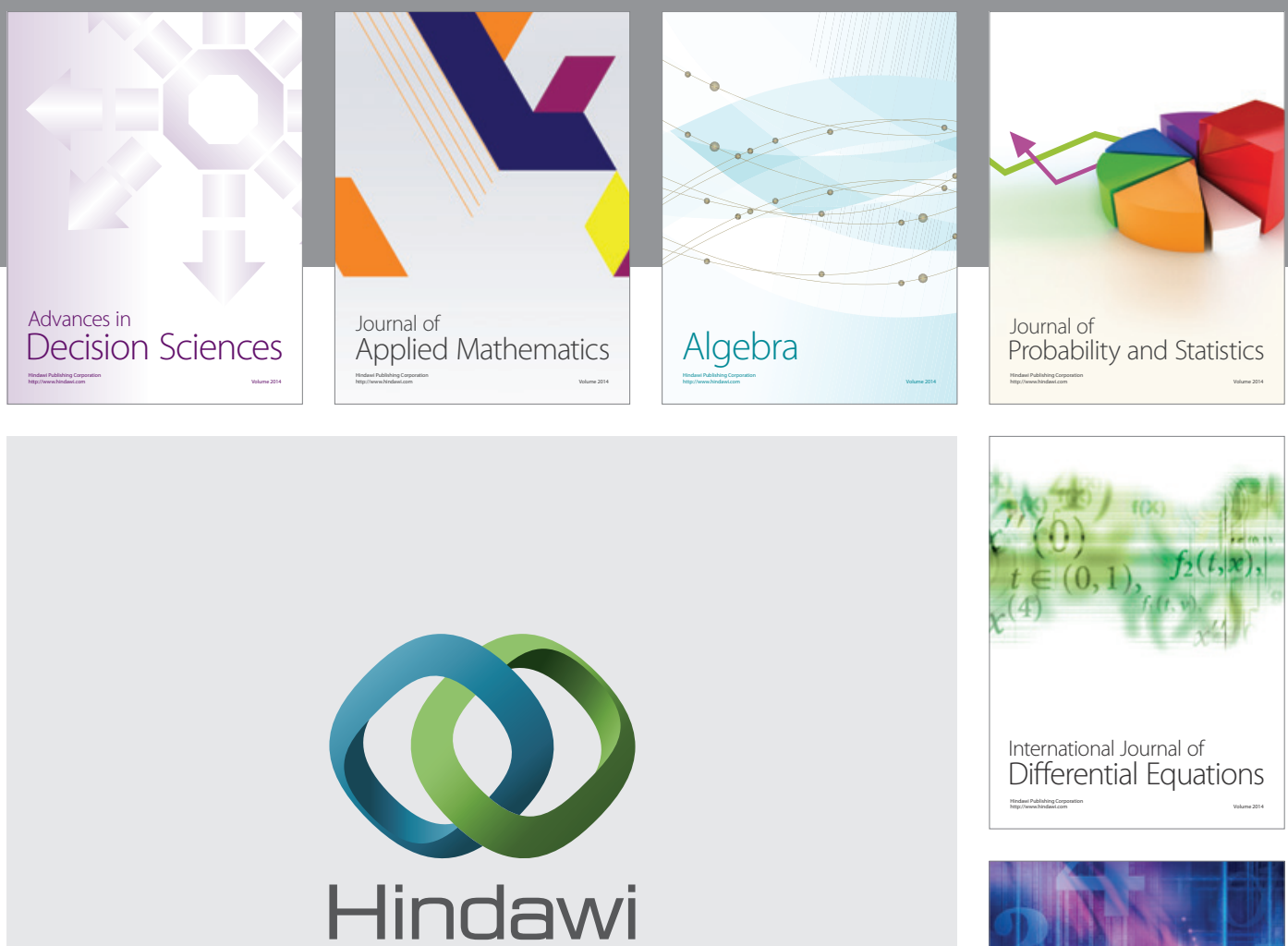

Submit your manuscripts at http://www.hindawi.com
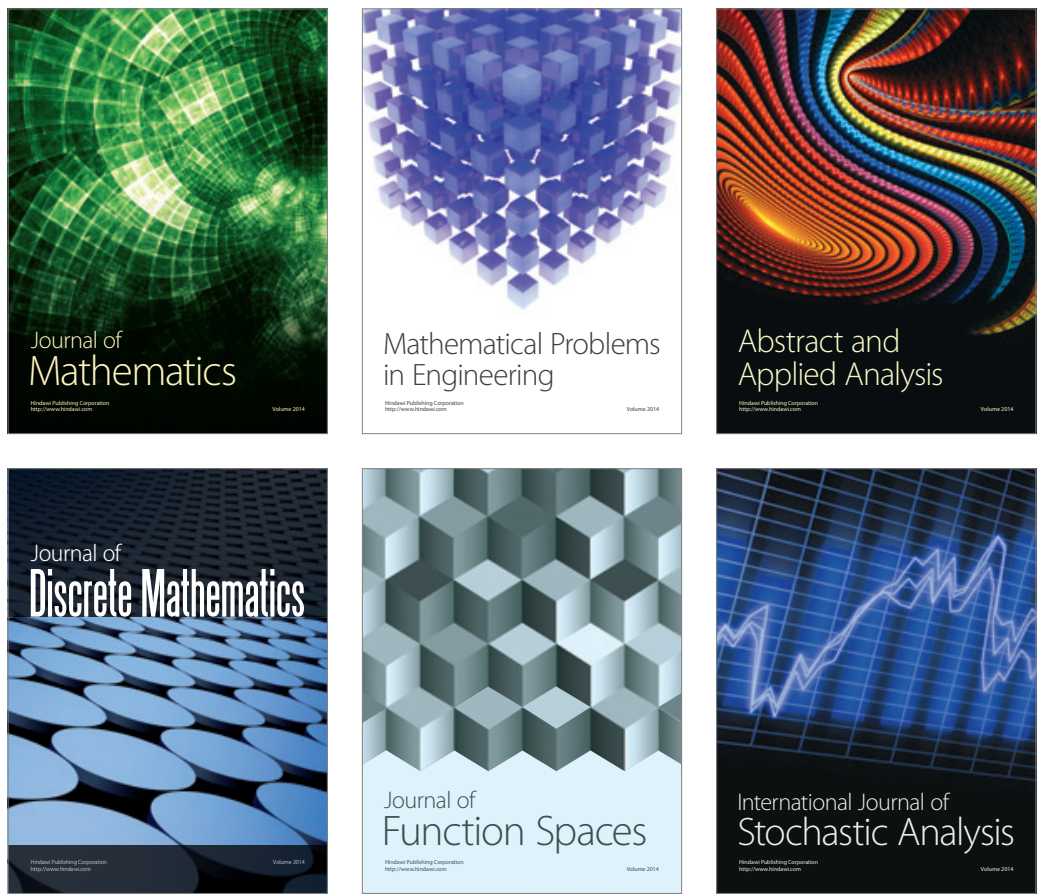

Journal of

Function Spaces

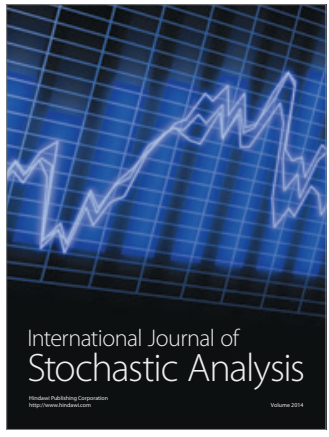

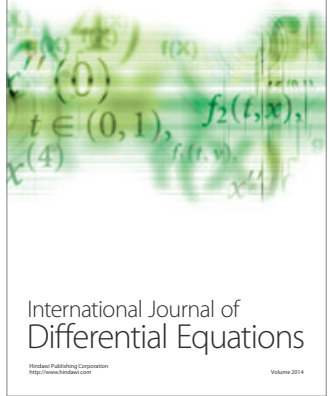
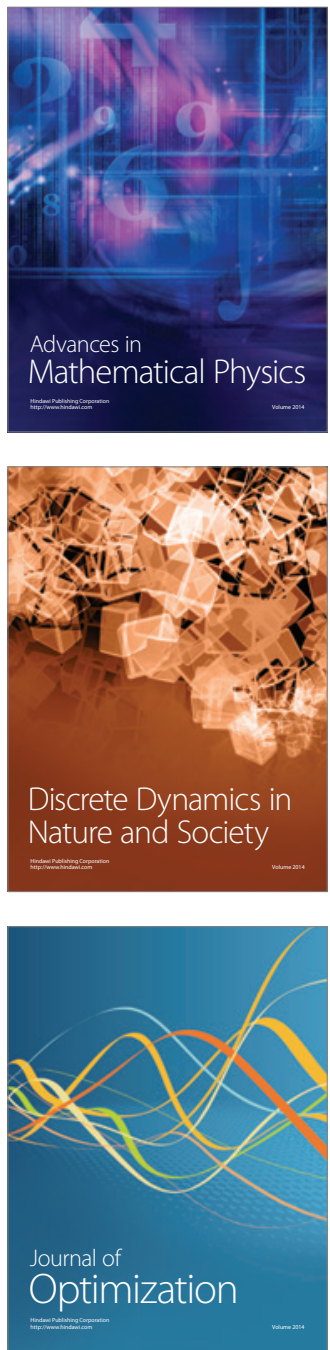